\title{
Physically-based Reduced-Order Capacity Loss Model for Graphite Anodes in Li-Ion Battery Cells tr
}

\author{
Xing Jin ${ }^{\mathrm{a}, *}$, Ashish Vora ${ }^{\mathrm{a}}$, Vaidehi Hoshing ${ }^{\mathrm{a}}$, Tridib Saha ${ }^{\mathrm{b}}$, Gregory Shaver ${ }^{\mathrm{a}}$, \\ R. Edwin García ${ }^{c}$, Oleg Wasynczuk ${ }^{b}$, Subbarao Varigonda ${ }^{\mathrm{d}}$ \\ ${ }^{a}$ School of Mechanical Engineering, Purdue University, 585 Purdue Mall, West Lafayette, \\ IN 47907 USA \\ ${ }^{b}$ School of Electrical and Computer Engineering, Purdue University, 465 Northwestern Ave. \\ West Lafayette, IN 47907 USA \\ ${ }^{c}$ School of Materials Engineering, Purdue University, 701 West Stadium Avenue West \\ Lafayette, IN 47907-2045 USA \\ ${ }^{d}$ Cummins Inc., Columbus, IN 47202 USA
}

\begin{abstract}
Physically-based Li-ion electrochemical cell models have been shown capable of predicting cell performance and degradation, but are computationally expensive for optimization-oriented design applications. Faster empirical models have been developed from experimental data, but are not generalizable to operating conditions outside of the range established by the calibration data. In this paper, a reduced-order capacity-loss model for graphite anodes is derived based upon the salient physical loss mechanisms to improve computational efficiency without sacrificing model fidelity. This model captures the two primary degradation mechanisms that occur in the graphite anode of a typical lithium ion cell: a) capacity loss due to Solid Electrolyte Interface (SEI) layer growth, and b) capacity loss due to isolation of active material. The model is calibrated and validated for a commercial 2.3-Ah cell with a Lithium Iron Phosphate (LFP) cathode and graphite anode. One data set is used for calibration, another four data sets are used for validation. The model matches experimental capacity degradation results within a $10 \%$ error. Moreover, the reported model is $2400 \times$ faster than currently existing more complex physically-based electrochemical
\end{abstract}

\footnotetext{
* Corresponding author

Email address: jin89@purdue.edu (Xing Jin)
}

Preprint submitted to Journal of ${ }^{A} T_{E} X$ Templates

September 13, 2016

(C) 2016. This manuscript version is made available under the Elsevier user license http://www.elsevier.com/open-access/userlicense/1.0/ 
models that are only slightly more accurate (less than $9 \%$ error).

Keywords: Reduced-order degradation model, Computationally efficient degradation model, Physically-based degradation model, Graphite/LiFePO 4 cell, Battery aging

2010 MSC: 00-01, 99-00

\section{Introduction}

For many years, rechargeable Li-ion batteries (LIBs) have been used in a wide variety of applications, ranging from portable electronics to electric vehicles [1]. Specifically, they have shown tremendous promise due to their high 5 specific energy and power densities compared to other existing battery technologies [2. These favorable properties, along with decreasing costs, have made LIBs the most attractive energy storage strategy for electric vehicle (EV) and hybrid electric vehicle (HEV) applications 3, 4. However, aging and degradation remain an impediment to widespread adoption.

Graphite is the most popular anode material for LIBs and its degradation is primarily a result of the growth of a Solid Electrolyte Interface (SEI) layer at the anode particles-electrolyte interface, and the fracture and isolation of the active material [5. The SEI layer is formed as a result of irreversible reactions between electrolyte and electrode materials (in particular, reduction of the organic elec-

15 trolyte solvent at the surface of the electrode) [6, 7, 8, The active material undergoes volumetric and structural changes during uptake and removal of lithium ions. These changes cause mechanical stress on particle structure resulting in cracks or structural damage, gradually isolating active materials (AM) during cycling [5, 9]. SEI layer growth and active material loss both result in a growth in internal resistance and reduction in the energy capacity of the cell.

Aging or degradation of the cell has been widely studied, and a wide variety of models exist in the literature, including physically-based electrochemical [5, 6, 10, 11], semi-empirical [12], and empirical models [3, 13]. Semiempirical and empirical models generally describe the dependence of battery 
resistance and capacity fade on various aging factors, including temperature, state of charge (SOC), C-rate, depth of discharge (DOD) [12 - in many cases through more-or-less arbitrary model constructs that enable a direct fit with experimental data. As a result, semi-empirical and empirical models are typically limited to operating conditions characteristic of the data used for calibration. In contrast, more complex electrochemical-based models, which better capture the underlying physical causes of the aging phenomena, are generally applicable to a larger range of operations conditions since they are based on fundamental physical principles. For example, Safari and coworkers developed a single-particle model to capture the SEI layer growth dynamics and active material loss rate in both electrode layers [5, 14. Here, graphite active material loss was modeled as a function of C-rate and temperature to achieve agreement with experimental data. Similarly, Prada et al. [10] outlined an isothermal physically-based aging model, in which the SEI layer growth is related to both capacity loss and internal resistance increase. However, each of these models require solving a complex charge conservation equations, etc.), which are computationally expensive (see Fig. 1(a) and Section 2).

To simplify the SEI layer growth model, several authors proposed purely diffusion limited models that assume the transport of the solvent across the SEI 45 layer to the surface of graphite particles is the limiting factor [6, 7]. Bazant and coworkers 8 ] went one step further and developed a constant-reaction-rate model for the SEI layer growth that accounts for both solvent diffusion through the SEI film as well as solvent reduction kinetics at the graphite surface.

In spite of the great deal of work, existing simplified models ignore the dependence of SEI formation rate on the state of charge (SOC) of the electrode, and the magnitude and direction of the intercalation current. In addition, existing models that include the spatial details and physical complexity in LIBs during long term use and degradation accumulation become impractical to use in technologically and commercially relevant settings because degradation occurs across long time scales, and the accessed computational time scales are 
unrealistic. In this paper, a mixed kinetic-diffusion controlled SEI layer growth sub-model is developed to capture the impact of both solvent diffusion and reduction kinetics. The SEI layer growth sub-model is combined with an active material loss sub-model to predict the capacity loss due to active material isolation. A commercial 2.3-Ah graphite/ $\mathrm{LiFePO}_{4}$ (LFP) cell is used as an example to calibrate and validate the model. This model is generalizable across a wide range of operating conditions, computationally efficient for model-based design, and simple enough for control algorithm development.

\section{Typical pseudo-2D electrochemical degradation model}

The inputs and outputs of a typical pseudo-2D electrochemical model (for instance, as implemented in AutoLion ST by EC Power [9]) are shown in Fig. 1] (a). Electrochemical models typically discretize the cathode and anode, defining a representative particle in each element. To calculate the loss in energy capacity of the battery requires numerically solving a set of partial differential 70 equations (PDEs) that govern the Li-ion transportation, synchronously with the constitutive laws that predict the degradation for each element.

\subsection{Capacity loss due to SEI layer growth}

For a generalized side reaction, let $\mathrm{S}$ represent all solvent reactants, that are reduced at the surface of the anode [11, 15]:

$$
\mathrm{S}+2 \mathrm{Li}^{+}+2 \mathrm{e}^{-} \rightleftarrows \mathrm{P}
$$

where $\mathrm{P}$ is the reduction product that forms the SEI layer. The rate of capacity loss due to SEI growth, $Q_{\mathrm{SEI}}$, is directly proportional to the rate of this side reaction, or equivalently the side reaction current density, $i_{s}$ :

$$
Q_{\mathrm{SEI}}=\int_{0}^{t} i_{s} d t
$$

The variables used in this paper are summarized in Table 1 
Table 1: Glossary of symbols

\begin{tabular}{|c|c|}
\hline \multicolumn{2}{|c|}{ Symbol Description } \\
\hline$t$ & Simulation time \\
\hline$i_{s, 0}$ & Exchange current density of the side reaction \\
\hline$i_{s}$ & Side reaction current \\
\hline$i_{0}$ & Exchange current density of the intercalation current \\
\hline$R$ & Ideal gas constant \\
\hline$T$ & Absolute temperature \\
\hline$n$ & The number of electrons reduced in the reaction \\
\hline$F$ & Faraday's constant \\
\hline$\alpha_{a}, \alpha_{c}$ & Anodic and cathodic transfer coefficients of electrochemical reaction \\
\hline$C_{\mathrm{S}}(0, t)$ & Concentration of the reactant at $x=0$ \\
\hline$C_{\mathrm{P}}(0, t)$ & Concentration of the reduction product $x=0$ \\
\hline$C_{\mathrm{Li}}(0, t)$ & Concentration of lithium ions at the interface of the graphite particle \\
\hline$C_{\mathrm{Li}, \max }$ & Maximum lithium concentration in the graphite electrode \\
\hline$C_{\mathrm{S}}^{*}$ & Bulk concentration of solvent reactant at equilibrium state \\
\hline$C_{\mathrm{P}}^{*}$ & Bulk concentration of reduction product at equilibrium state \\
\hline$C_{\mathrm{Li}}^{*}$ & Bulk lithium concentration in the graphite electrode \\
\hline$C_{e}$ & Li-ion concentration in the electrolyte \\
\hline$\eta_{s}$ & Overpotential of the side reaction \\
\hline$\eta_{k}$ & Kinetic overpotential \\
\hline$E^{0^{\prime}}$ & Formal potential of the anode \\
\hline$E_{e q}$ & Equilibrium potential of the anode \\
\hline$\phi_{s}$ & Electric potential in the solid phase \\
\hline$\phi_{e}$ & Electric potential in the electrolyte phase \\
\hline$R_{\mathrm{SEI}}$ & Resistance due to the SEI layer \\
\hline$j$ & Li-ion insertion/de-insertion current density \\
\hline$k_{s, a}, k_{s, c}$ & Rate constant for the anodic and cathodic side reaction \\
\hline$k_{s}$ & Side reaction constant (fitting parameter) \\
\hline$U_{s}^{\mathrm{OCP}}$ & Open circuit potential of the side reaction \\
\hline$U_{n}^{\mathrm{OCP}}$ & Open circuit potential of the anode \\
\hline$\varepsilon_{\mathrm{AM}}$ & Volume fraction of active material \\
\hline$\varepsilon_{\mathrm{AM}, 0}$ & Initial volume fraction of active material \\
\hline$V_{r}$ & Volume of $\mathrm{r}^{t h}$ element in anode \\
\hline$V$ & Total volume of the anode \\
\hline$D_{\mathrm{S}}$ & Diffusion coefficients of the reactant \\
\hline$D_{\mathrm{P}}$ & Diffusion coefficients of the reduction product \\
\hline$D_{\mathrm{S}}^{0}$ & Pre-exponential factor for $\mathrm{S}$ diffusion \\
\hline$E_{\mathrm{S}}$ & Activation energy for the $\mathrm{S}$ diffusion process \\
\hline$k_{\mathrm{AM}}^{o}$ & Pre-exponential factor for active material loss \\
\hline$E_{\mathrm{AM}}$ & Activation energy of the active material loss \\
\hline$R_{s}$ & Particle radius of the active material \\
\hline$A$ & Active surface area of the anode \\
\hline$a_{s}$ & Specific surface area of the anode \\
\hline
\end{tabular}




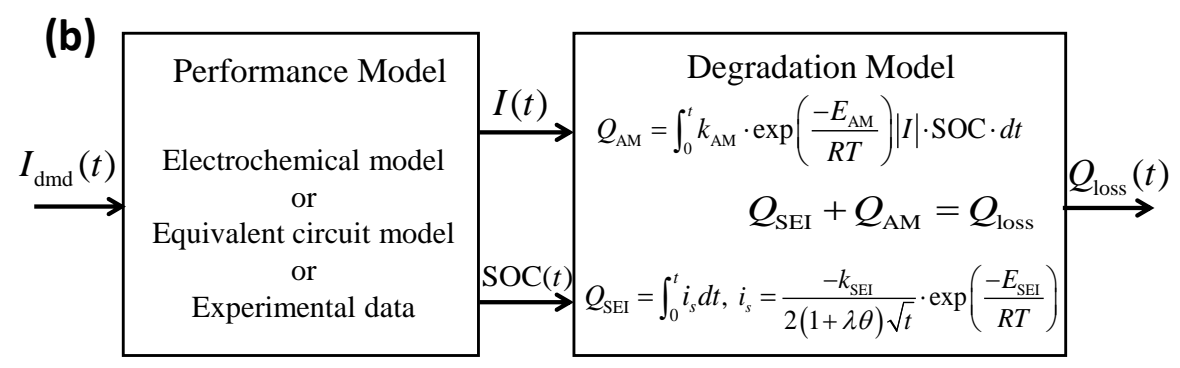

Figure 1: Schematic diagram of the degradation model input and output. (a) is a typical electrochemical model [9]. Equations (i) and (ii) represent the conservation of charge in the solide phase and electrolyte phase, (iii) gives the species conservation in the electrolyte, (iv) represents the Li-ion diffusion in active material particle, and (v) is the Butler-Volmer equation. (b) is the simplified reduced-order degradation model proposed herein.

A schematic of the electrode particle-electrolyte interface is shown in Fig. 2 so For an SEI layer much smaller in thickness than a typical particle of active material, the particle surface can be assumed to have zero curvature.

In the pseudo-2D electrochemical system, the rate of SEI formation is described by a Bulter-Volmer kinetics relation [11, 15]:

$$
i_{s}(t)=i_{s, 0}\left[\frac{C_{\mathrm{P}}(0, t)}{C_{\mathrm{P}}^{*}} \exp \left(\frac{\alpha_{a} n F}{R T} \eta_{s}\right)-\frac{C_{\mathrm{S}}(0, t)}{C_{\mathrm{S}}^{*}}\left(\frac{C_{\mathrm{Li}}(0, t)}{C_{\mathrm{Li}}^{*}}\right)^{2} \exp \left(-\frac{\alpha_{c} n F}{R T} \eta_{s}\right)\right]
$$


Figure 2: Schematic diagram of the SEI layer formation on the graphite electrode. The side reaction occurs at the interface of the active material and SEI layer. $\mathrm{S}$ is the solvent reactant, and $\mathrm{P}$ is the reduction product that forms the SEI layer. $i_{s}(t)$ is the side reaction current, $j(t)$ is the flux of Li-ions insertion/de-insertion (i.e. actual current passing through the cell), $C_{\mathrm{S}}^{*}$ is the bulk concentration of the reactant in electrolyte, $C_{\mathrm{S}}(0, t)$ is the concentration of reactant at $x=0, \varepsilon_{\mathrm{AM}}$ is the volume fraction of active material, and $z(t)$ is the is the nominal concentration of Li ions in the graphite particle (i.e. a measure of SOC). Dotted black lines show the diffusion of the Li-ions, solvent reactant $\mathrm{S}$, and reduction product, $\mathrm{P}$.

where $i_{s, 0}=n F k_{s, c}^{\alpha_{a}} k_{s, a}^{\alpha_{c}}\left(C_{\mathrm{P}}^{*}\right)^{\alpha_{c}}\left(C_{\mathrm{S}}^{*}\right)^{\alpha_{a}}\left(C_{\mathrm{Li}}^{*}\right)^{2 \alpha_{a}}$, and $\eta_{s}$ is the overpotential

$$
\eta_{s}=\eta_{k}+U_{n}^{\mathrm{OCP}}-U_{s}^{\mathrm{OCP}}
$$

where $U_{s}^{\mathrm{OCP}}=0.4 \mathrm{~V}$ is the reversible open circuit potential (with respect to a pure Li electrode) for solvent reduction [4, 11, and $U_{n}^{\mathrm{OCP}}$ is the open circuit potential of the anode, which is a function of SOC [15], as given in the Appendix A From Fig. 1 (a) and 9], the kinetic overpotential $\eta_{k}$ is:

$$
\eta_{k}=\phi_{s}-\phi_{e}-U_{n}^{\mathrm{OCP}}-R_{\mathrm{SEI}} j
$$

If the solvent reaction is irreversible, then the first term of Eq. (3) on the 
right-hand side is effectively equal zero. Similarly, if diffusion of $\mathrm{S}$ through the SEI layer is the limiting factor for the side reaction to occur, then the diffusion of Li-ions can be ignored, and $C_{\mathrm{Li}}(0, t)$ equals the bulk concentration of Li-ions in the active material, $C_{\mathrm{Li}}^{*}$. Thus:

$$
i_{s}=-n F k_{s} C_{\mathrm{S}}(0, t) \exp \left(-\frac{\alpha_{c} n F}{R T} \eta_{s}\right)
$$

85 in agreement with [4, 9, 14. The concentration of $\mathrm{S}$ across the SEI layer, $C_{\mathrm{S}}(x, t)$, is described by Eq. (7) [4, 9, 14].

$$
\frac{\partial C_{\mathrm{S}}(x, t)}{\partial t}=D_{\mathrm{S}} \frac{\partial^{2} C_{\mathrm{S}}(x, t)}{\partial x^{2}}
$$

\subsection{Capacity loss due to active material loss}

The active material undergoes volume and structural changes during current cycling. These changes cause mechanical stress resulting in structural damage,

9o gradually isolating the active material (AM). The rate of lithium loss is:

$$
\frac{d Q_{\mathrm{Li}, \mathrm{loss}}(r, t)}{d t}=\frac{d \varepsilon_{\mathrm{AM}}(r)}{d t} \cdot \mathrm{SOC} \cdot V_{r} \cdot C_{\mathrm{Li}, \max }
$$

where $r$ represents the $r^{t h}$ representative particle, and the active material loss rate is defined herein as 9 :

$$
\frac{d \varepsilon_{\mathrm{AM}}(r)}{d t}=-k(T)|j(r, t)|
$$

where $k(T)$ is a temperature-dependent rate coefficient, which is typically described by an Arrhenius equation [6, 9, 10]:

$$
k(T)=k_{\mathrm{AM}}^{o} \exp \left(\frac{-E_{\mathrm{AM}}}{R T}\right)
$$

The integral of Eq. 8 across the anode results in:

$$
Q_{\mathrm{Li}, \mathrm{loss}}=\sum_{r} \int_{0}^{t} k(T)|j(r, t)| \cdot \mathrm{SOC} \cdot V_{r} \cdot C_{\mathrm{Li}, \max } d t
$$




\section{Reduced-order degradation model development}

The inputs and outputs of the proposed degradation model are shown in

${ }_{95}$ Fig. 1 (b). The inputs to this model are the cell current vs time, $I(t)$, and the cell SOC vs time, $\operatorname{SOC}(t)$. These inputs can be determined in one of several ways, including: 1) an electrochemical model, 2) an equivalent circuit model, or 3) experimental data. The output of the degradation model is the loss in energy capacity of the battery, $Q_{\text {loss }}$.

\subsection{Capacity loss due to SEI layer growth}

Instead of numerically solving for $\eta_{k}$ and $C_{\mathrm{S}}(0, t)$ on an element-by-element basis, a mixed kinetic-diffusion control SEI layer growth model based on single particle model is developed in this section.

Similar to the assumption made by [4, 14, 9, it is assumed that the diffusion of the solvent reactant, S, through the SEI layer is the limiting factor for the side reaction. Thus, the Li-ions diffusion process can be ignored, and the diffusion of the reactant, S, and reduction product, $\mathrm{P}$, are described by Eqs. (7) and (12),

$$
\frac{\partial C_{\mathrm{P}}(x, t)}{\partial t}=D_{\mathrm{P}} \frac{\partial^{2} C_{\mathrm{P}}(x, t)}{\partial x^{2}}
$$

The initial conditions are $C_{\mathrm{S}}(x, 0)=C_{\mathrm{S}}^{*}, C_{\mathrm{P}}(x, 0)=0$, express the homogeneity of the solution at $t=0$. The semi-infinite boundary conditions are $\lim _{x \rightarrow \infty} C_{\mathrm{S}}(x, t)=C_{\mathrm{S}}^{*}, \lim _{x \rightarrow \infty} C_{\mathrm{P}}(x, t)=0$, specify the bulk far field concentrations in the electrolyte. The charge flux density boundary conditions are:

$$
\begin{gathered}
-D_{\mathrm{S}}\left(\frac{\partial C_{\mathrm{S}}(x, t)}{\partial x}\right)_{x=0}=\frac{i_{s}}{n F} \\
D_{\mathrm{S}}\left(\frac{\partial C_{\mathrm{S}}(x, t)}{\partial x}\right)_{x=0}=-D_{\mathrm{P}}\left(\frac{\partial C_{\mathrm{P}}(x, t)}{\partial x}\right)_{x=0}
\end{gathered}
$$

Equations (7) and (12) lead to (detailed derivation in Appendix B 16]):

$$
i_{s}(t)=-\frac{n F C_{\mathrm{S}}^{*} \sqrt{D_{\mathrm{S}}}}{\sqrt{\pi t}(1+\lambda \theta)}
$$


where $\lambda=\frac{C_{\mathrm{S}}^{*} \sqrt{D_{\mathrm{S}}}}{C_{\mathrm{P}}^{*} \sqrt{D_{\mathrm{P}}}}$, and $\theta$ is (see Appendix B:

$$
\theta=\frac{C_{\mathrm{S}}(0, t)}{C_{\mathrm{P}}(0, t)}=\exp \left[\frac{n F}{R T}\left(\eta_{k}+U_{n}^{\mathrm{OCP}}-U_{s}^{\mathrm{OCP}}\right)\right]
$$

Capacity loss due to SEI layer growth is computed as follows:

$$
Q_{\mathrm{SEI}}=\int_{0}^{t} i_{s} d t=\int_{0}^{t}-\frac{n F C_{\mathrm{S}}^{*} \sqrt{D_{\mathrm{S}}}}{\sqrt{\pi t}(1+\lambda \theta)} d t
$$

To compute $\theta$, it is necessary to first compute $\eta_{k}$. From Fig. 1 (b) and 115

Eq. (5), computing $\eta_{k}$ requires numerically solving several PDEs. An alternate approach, suggested by [10, 17, is used to estimate $\eta_{k}$ :

$$
\begin{gathered}
\eta_{k}=\frac{R T}{\alpha F} \ln \left(\xi+\sqrt{\xi^{2}+1}\right) \\
\xi=\frac{R_{s} I}{6 \varepsilon_{\mathrm{AM}, 0} i_{0} V} \\
i_{0}=k_{0} C_{e}^{{ }^{\alpha_{a}}}\left(C_{\mathrm{Li}, \max }-C_{\mathrm{Li}}\right)^{\alpha_{a}} C_{\mathrm{Li}}{ }^{\alpha_{c}}
\end{gathered}
$$

$i_{0}$ is set as a constant in agreement with [10, 17, 18].

According to [6], the diffusion coefficient $D_{\mathrm{S}}$ in Eq. (17) is given by:

$$
D_{\mathrm{S}}=D_{\mathrm{S}}^{0} \exp \left(-\frac{E_{\mathrm{S}}}{R T}\right)
$$

Substitution of Eq. (21) into Eq. (15) leads to:

$$
i_{s}(t)=-\frac{k_{\mathrm{SEI}} \exp \left(-\frac{E_{\mathrm{SEI}}}{R T}\right)}{2(1+\lambda \theta) \sqrt{t}}
$$

where

$$
k_{\mathrm{SEI}}=\frac{2 n F C_{\mathrm{S}}^{*} \sqrt{D_{\mathrm{S}}^{0}}}{\sqrt{\pi}}
$$

and

$$
E_{\mathrm{SEI}}=\frac{E_{\mathrm{S}}}{2}
$$

Thus, the capacity loss due to SEI layer growth becomes:

$$
Q_{\mathrm{SEI}}=\int_{0}^{t} i_{s} d t=\int_{0}^{t}-\frac{k_{\mathrm{SEI}} \exp \left(-\frac{E_{\mathrm{SEI}}}{R T}\right)}{2(1+\lambda \theta) \sqrt{t}} d t
$$



135

To the best of the authors' knowledge, this is the first study to develop a mixed kinetic-diffusion controlled analytic model to capture the impact of both solvent diffusion and reduction kinetics for SEI layer growth and the associated capacity loss. This reduced-order model results from the analytic solution of constitutive laws that govern the side reaction, i.e. reduction kinetics, diffusion of solvent, $\mathrm{S}$, and reduction product, $\mathrm{P}$, and it includes no ad-hoc terms. It is a simple, computationally efficient expression with only three fitted parameters, and capable of predicting the capacity loss due to SEI growth accurately across a wide range of temperatures and SOCs.

Special Case 1- Diffusion limited degradation model. If the side reaction is only limited by diffusion (in agreement with [6]), then $C_{\mathrm{S}}(0, t)$ is negligible and $\theta=$ $\frac{C_{\mathrm{S}}(0, t)}{C_{\mathrm{P}}(0, t)} \rightarrow 0$. Equations 22 and $\sqrt{23}$ are simplified as:

$$
\begin{aligned}
i_{s}(t) & =-\frac{k_{\mathrm{SEI}} \exp \left(-\frac{E_{\mathrm{SEI}}}{R T}\right)}{2 \sqrt{t}} \\
Q_{\mathrm{SEI}} & =k_{\mathrm{SEI}} \exp \left(-\frac{E_{\mathrm{SEI}}}{R T}\right) \sqrt{t}
\end{aligned}
$$

which shows dependency on square root of time and is thus sometimes referred to calendar-driven capacity loss [6, 7, 8]. However, because the solution is independent on the kinetics or the SOC and current of the cell, the capacity loss prediction percentage error is as high as 50\%, as shown later in Sections 4 and 5 .

Special Case 2- Zero current density model. If the cell is is simply stored away (i.e., constant SOC), then from Eqs. 16 and (18), the capacity loss is:

$$
Q_{\mathrm{SEI}}=\frac{k_{\mathrm{SEI}} \exp \left(-\frac{E_{\mathrm{SEI}}}{R T}\right) \sqrt{t}}{(1+\lambda \theta)}
$$

Equation (26) also delivers a square root of time dependency, as shown in literature 6 , 7, 8, This special case is useful in calibrating the SEI layer growth sub-model, as will be demonstrated in Section 4 


$$
Q_{\mathrm{Li}, \text { loss }}=\int_{0}^{t} k(T)|j(t)| \cdot \mathrm{SOC} \cdot V \cdot C_{\mathrm{Li}, \max } d t
$$

where $k(T)$ is the same as defined in Eq. 10$)$, and $j(t)$ is the volumetric current density:

$$
j(t)=\frac{I}{A} a_{s}
$$

Before the active material loss occurs, the total amount of usable lithium ions within the active material of the electrode is:

$$
Q_{\mathrm{Li}, 0}=\varepsilon_{\mathrm{AM}, 0} \cdot V \cdot C_{\mathrm{Li}, \max }
$$

As such, the capacity loss (in percentage) due to active material loss is

$$
Q_{\mathrm{AM}}=\frac{Q_{\mathrm{Li}, \text { loss }}}{Q_{\mathrm{Li}, 0}}=\int_{0}^{t} k_{\mathrm{AM}} \exp \left(\frac{-E_{\mathrm{AM}}}{R T}\right) \cdot \mathrm{SOC} \cdot|I| d t
$$

where $k_{\mathrm{AM}}=\frac{k_{\mathrm{AM}}^{o} a_{s}}{A \varepsilon_{\mathrm{AM}, 0}}$.

At constant temperature, Eq. 30 reduces to:

$$
Q_{\mathrm{AM}}=k_{\mathrm{AM}} \exp \left(\frac{-E_{\mathrm{AM}}}{R T}\right) \cdot A h_{\mathrm{SOC}}
$$

where $A h_{\mathrm{SOC}}=\int_{0}^{t} \mathrm{SOC} \cdot|I| d t$ is the SOC-weighted ampere-hour throughput.

\subsection{Combined capacity loss model summary}

In summary, a reduced-order capacity loss model has been developed based on salient physical loss mechanisms. This model differs from computationally 
efficient semi-empirical and empirical models as it results from the analytic solution of widely accepted constitutive laws, and includes no ad-hoc terms. More complex electrochemical models couple these same constitutive laws with Li-ion transport PDEs, and as such, capacity loss prediction using those models is computationally expensive. Unlike the electrochemical models, the reducedorder degradation model proposed in this effort only requires lumped input information, current vs time and SOC vs time, to predict the capacity loss. The total capacity loss (in percentage) can be quantified as:

$$
Q_{\mathrm{loss}}=Q_{\mathrm{SEI}}+Q_{\mathrm{AM}}
$$

where $Q_{\mathrm{SEI}}$ is given by Eq. $(23)$, and $Q_{\mathrm{AM}}$ is given by Eq. $(30)$. The detailed sub-models are shown in Table 2 This physically-based, generalizable, reducedorder degradation model is nearly as accurate as more complex electrochemical models, while enabling a 2400 times speedup, as will be shown in Section 5 .

Computational savings vs pseudo-2D electrochemical model. In the electrochemical model, to calculate the capacity loss due to SEI layer growth and active material loss, it is necessary to numerically solve for the side reaction overpotential, solvent reactant concentration, and current density, resolved across the thickness of the electrode. This requires solving the governing PDEs of the electrochemical performance model (and solving them multiple times if using a multi-element model). The reduced-order degradation model, however, only requires lumped input information, specifically, current vs time and SOC vs time, from the performance model. This allows the use of simpler equivalent circuit models - for prediction of $I(t)$ and $\operatorname{SOC}(t)$ - that can run approximately $80 \mathrm{x}$ faster than an electrochemical performance model [such as that implemented in AutoLion ST (ALST)].

If the current demand on the battery is assumed to be captured in a representative cycle (e.g. a vehicle driven over a representative drivecycle), the performance model can be executed for only one cycle and the resulting SOC vs time data can be recorded. Then these current vs time and SOC vs time profiles 
Table 2: Reduced-order degradation model

\begin{tabular}{ll}
\hline$Q_{\mathrm{SEI}}$ & $Q_{\mathrm{SEI}}=\int_{0}^{t}-\frac{k_{\mathrm{SEI}} \exp \left(-\frac{E_{\mathrm{SEI}}}{R T}\right)}{2(1+\lambda \theta) \sqrt{t}} d t$ \\
\hline$\theta$ & $\theta=\exp \left[\frac{n F}{R T}\left(\eta_{k}+U_{n}^{\mathrm{OCP}}-U_{s}^{\mathrm{OCP}}\right)\right]$ \\
\hline$\eta_{k}$ & $\eta_{k}=\frac{R T}{\alpha F} \ln \left(\xi+\sqrt{\xi^{2}+1}\right)$ \\
\hline$\xi$ & $\xi=\frac{R_{s} I}{6 \varepsilon_{\mathrm{AM}, 0} i_{0} V}$ \\
\hline$U_{n}^{\mathrm{OCP}}$ & $-\frac{0.0172}{z}+\frac{0.0019}{z^{1.5}}+0.0299 z^{1 / 2}$ \\
\hline$U_{s}^{\mathrm{OCP}}$ & $-0.7984 \exp (0.4465 z-0.4108), z=\mathrm{SOC}$ \\
\hline$Q_{\mathrm{AM}}$ & $U_{s}^{\mathrm{OCP}}=0.4$ \\
\hline Total capacity loss & $Q_{\mathrm{AM}}=\int_{0}^{t} k_{\mathrm{AM}} \exp \left(\frac{-E_{\mathrm{AM}}}{R T}\right) \cdot \mathrm{SOC} \cdot|I| d t$ \\
\hline
\end{tabular}

Table 3: Key parameters of the 2.3-Ah 26650 LFP cell used in the simulation

\begin{tabular}{ccl}
\hline Parameters & Value & unit \\
\hline$R_{s}$ & $7.5 \times 10^{-6}$ & $\mathrm{~m}$ \\
$V$ & $1.26 \times 10^{-5}$ & $\mathrm{~m}^{3}$ \\
$i_{0}$ & 0.05 & $\mathrm{~A} / \mathrm{m}^{2}$ \\
$\varepsilon_{\mathrm{AM}, 0}$ & 0.552 & \\
$\alpha$ & 0.5 & \\
\hline
\end{tabular}


can be repeated with the desired idle time between cycles, and fed as inputs to

the degradation model. This allows the degradation model to be run through end-of-life, but the performance model only has to be executed over one cycle. Using this approach, in simulations run for this study, the reduced-order model enabled a 2400 times speedup in simulations through end-of-life, as compared to the pseudo-2D electrochemical model (implemented in ALST), without compromising prediction accuracy (details in Section 5). This is a significant benefit, especially for use in large-scale system-level simulation studies.

\section{Simplified reduced-order model calibration}

The degradation model is derived, calibrated and validated with a focus on the graphite anode and is assumed to be independent of the cathode material used. Data from a 2.3 Ah graphite/LFP cell (Manufacture: A123 Systems) is used to validate the model simplification strategy outlined in this paper for two reasons: 1) Availability of published experimental data for calibration and model validation due to relative maturity of this battery chemistry [3, 5, 20, 21, 22, 2) For cells with an LFP cathode and graphite anode, the degradation mechanisms in the cathode can be neglected [5, 21] and, as such, the capacity loss measured through experiments can be fully attributed to anode degradation.

As suggested by Delacourt and coworkers [5, the SEI layer growth submodel is first calibrated using experimental data from aging tests under storage conditions. Experimental data from aging tests under cycling conditions is then used to calibrate the active material loss sub-model.

There are five fitted parameters in this simplified reduced-order model, including $k_{\mathrm{SEI}}, E_{\mathrm{SEI}}, \lambda, k_{\mathrm{AM}}$, and $E_{\mathrm{AM}}$. The three parameters in the SEI-layer growth sub-model $\left(k_{\mathrm{SEI}}, E_{\mathrm{SEI}}\right.$, and $\left.\lambda\right)$ are tuned using experimental data from cell aging tests under storage conditions [5]. The two parameters in the active material loss sub-model $\left(k_{\mathrm{AM}}, E_{\mathrm{AM}}\right)$ are tuned using the active material loss experimental data from cycling tests [5]. All of the fitted parameters are listed in Table 4 . 
Table 4: Coefficients of the simplified reduced-order model

\begin{tabular}{ccl}
\hline Fitted parameters & Value & unit \\
\hline$k_{\text {SEI }}$ & 72.53 & $1 / \mathrm{sec}^{1 / 2}$ \\
$E_{\text {SEI }}$ & 39398 & $\mathrm{~J} / \mathrm{mol}$ \\
$k_{\mathrm{AM}}$ & 0.0137 & $1 / \mathrm{Ah}$ \\
$E_{\mathrm{AM}}$ & 39500 & $\mathrm{~J} / \mathrm{mol}$ \\
$\lambda$ & $4.77 \times 10^{-5}$ & \\
\hline
\end{tabular}

After calibration, Eq. 23) is used to predict the capacity fade due to SEI layer growth for other SOCs and temperatures. The experimental data (circles,

210 dashed lines) for a 2.3 Ah LFP cell are shown in Fig. 3 under different storage conditions, demonstrating very good agreement. A diffusion limited model (Eq. (25)) was also calibrated to minimize the error across the available experimental data. Due to the lack of SOC information in a diffusion limited model, the capacity loss prediction is the same regardless of SOC (magenta line) and a large discrepancy (percentage error is as high as 50\%) is observed at very high and very low SOC conditions.

The active material volume fraction experimental data [5] and the predicted value from $Q_{\mathrm{Am}}$ under different temperatures are shown in Fig. $4 k_{\mathrm{Am}}$ and 220 of the active material matches well with the experimental data at $25^{\circ} \mathrm{C}$. At $45^{\circ} \mathrm{C}$, the simulation result correlates well with the experimental data unless the last point. Since the active material volume fraction was calculated based on the differential-voltage method in [5], the discrepancy of the last point may be the 225 result of measurement noise in voltage and capacity, or some non-uniformity in the electrode material. 


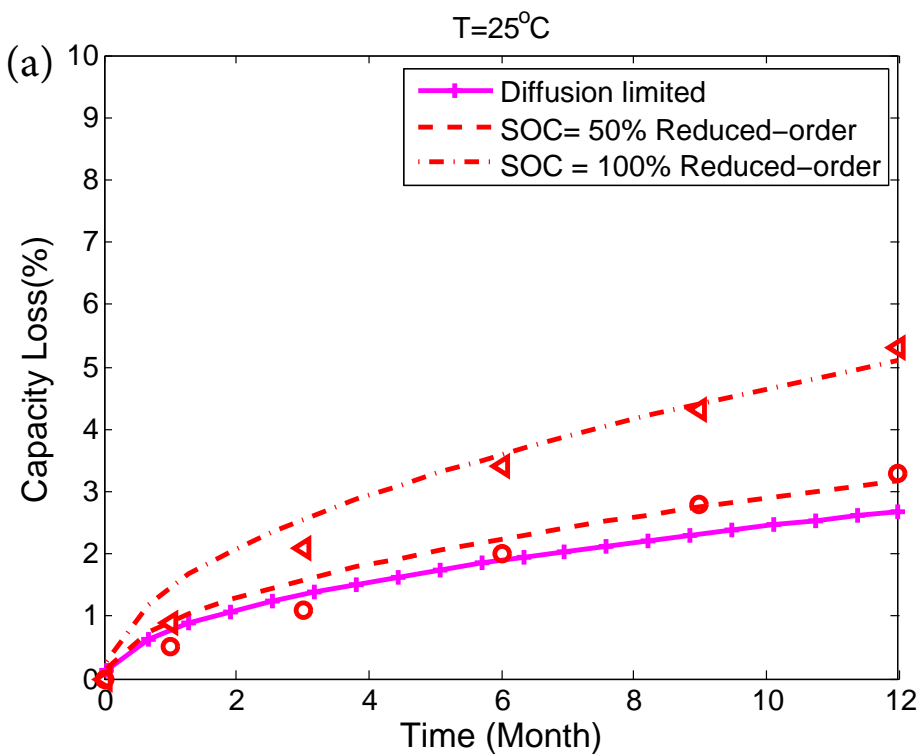

$\mathrm{T}=45^{\circ} \mathrm{C}$

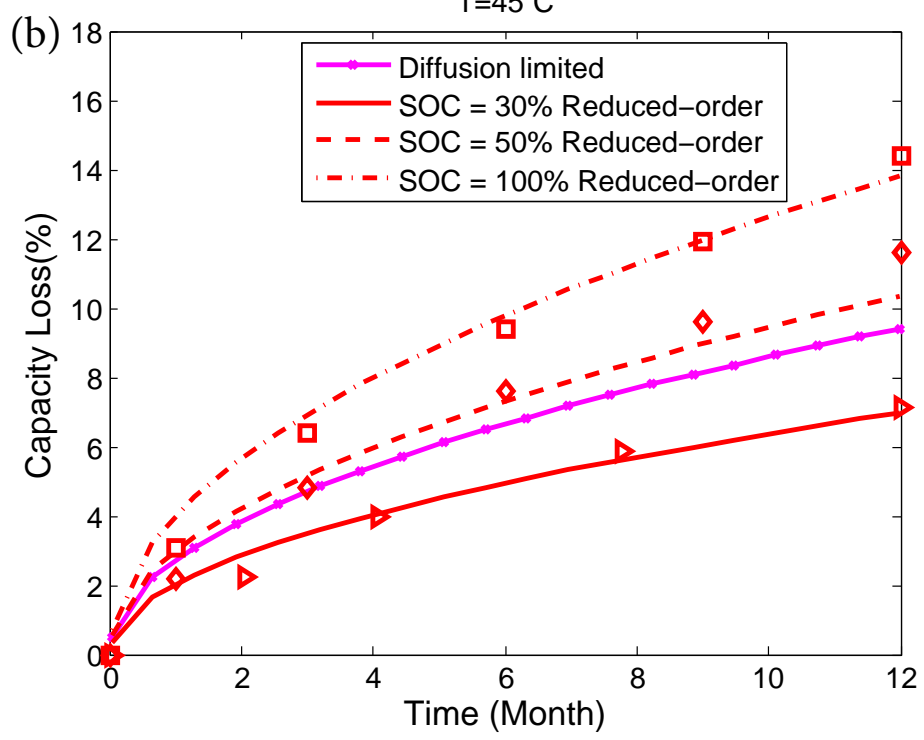

Figure 3: Calibration of SEI layer growth sub-model during storage conditions [5]. (a) Results at $25^{\circ} \mathrm{C}$. Circles represent experimental data of $50 \%$ SOC, left pointing triangles represent experimental data of $100 \%$ SOC. (b) Results at $45^{\circ} \mathrm{C}$. Right pointing triangles represent experimental data of $30 \%$ SOC, diamonds represent experimental data of $50 \%$ SOC, and squares represent experimental data of $100 \%$ SOC. 


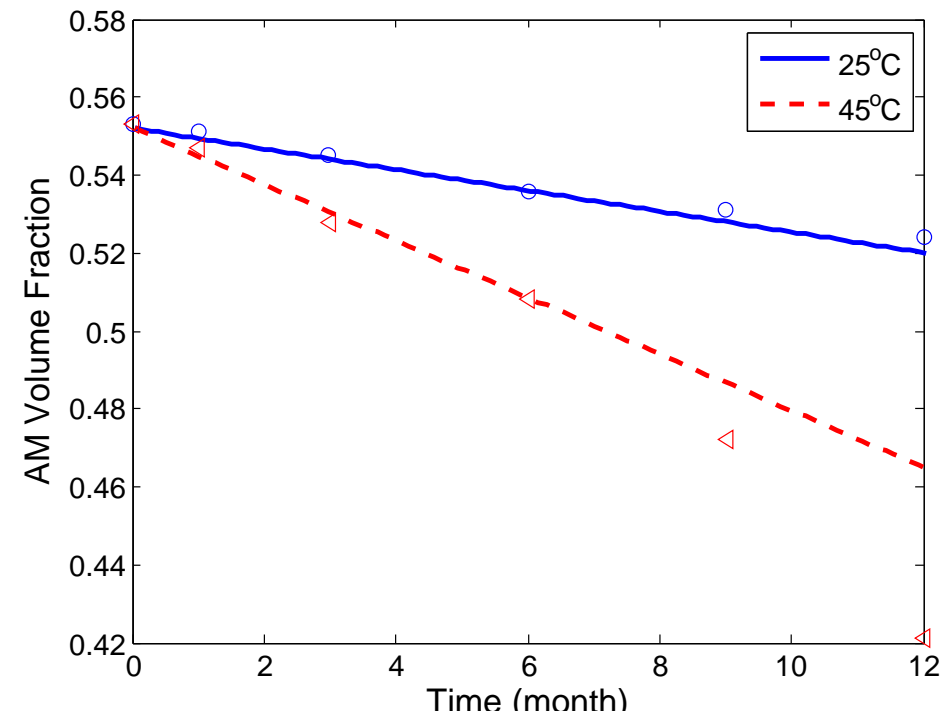

Figure 4: Calibration results for the active material loss sub-model, Eq. 31. Circles and triangles are experimental data at $25^{\circ} \mathrm{C}$ and $45^{\circ} \mathrm{C}$ respectively [5]. Solid/dashed lines are simulation results after model calibration. Cell cycling was simulated with a CC-CV charging protocol and a $1 \mathrm{C}$ constant current discharge.

\section{Simplified reduced-order model validation}

The reduced-order degradation model and electrochemical model are validated against four experimental data sets $3,20,21,22$, that represent constant C-rate charge/discharge (low C-rate and high C-rate) and variable C-rate dynamic profiles representative of EV and HEV use cases. Repeated current vs time profiles from the experimental datasets are imposed as an input to the pseudo-2D electrochemical model. The SOC vs time data output from the first cycle of the pseudo-2D electrochemical model simulation is used along with the experimental current vs time profile for input to the reduced-order degradation model. A third model that uses the diffusion limited SEI layer growth submodel (Equation 25 with $k_{\mathrm{SEI}}=2306.9$ and $\left.E_{\mathrm{SEI}}=49569\right)$ and the simplified active material loss sub-model (Equation (31)) is also shown.

To quantify the difference between the experimental data and the simulation results of the three models, percentage error is defined as the per- 
centage difference of the capacity loss at the ending point of the experiment: percentage error $=\frac{\left|Q_{\exp }-Q_{\text {loss }}\right|}{Q_{\exp }} \times 100 \%$, where $Q_{\text {loss }}$ is the capacity loss predicted by the models, and $Q_{\text {exp }}$ is the capacity loss of the experimental data set at the end of testing.

\subsection{Experimental data set 1 - High C-rate cycling}

The simplified reduced-order, pseudo-2D electrochemical, and diffusion limited models show good correlation with the experimental data, as shown in Fig. 5 (c). This data set is consistent with high $\mathrm{C}$ rates (up to $16 \mathrm{C}$ ) that occur in heavy vehicles during braking and acceleration maneuvers [22]. The diffusion limited model matches well with the experimental data because the average SOC (50\%) for this specific drive cycle, as shown in Fig. 5 (b), is very close to the average SOC of the data $(\sim 45 \%)$ used to calibrate the diffusion limited model.

The capacity loss due to SEI layer growth, $\mathrm{Q}_{\mathrm{SEI}}$, and active material loss, ${ }_{255} \mathrm{Q}_{\mathrm{AM}}$, predicted by the reduced-order, diffusion limited, and pseudo-2D electrochemical models is shown in Fig. 5 (d). Overall, all three models perform similarly. In this high C-rate case, the capacity loss due to active material loss, $\mathrm{Q}_{\mathrm{AM}}$, is $\sim 3$ times larger than that due to SEI layer growth, $\mathrm{Q}_{\mathrm{SEI}}$. However, the magnitude of $\mathrm{Q}_{\mathrm{SEI}}$ is significant enough that it has an impact on total capacity loss. As such, these results support the use of a degradation model that predicts capacity loss from both SEI layer growth and AM loss.

\subsection{Experimental data set 2 - Representative of HEV duty cycle}

The capacity loss predicted by each of the three models is shown in Fig. 6 (c). All three models show good agreement with the experimental data [20], which is obtained by cycling the cell with a synthetic current profile, created such that the aging factors i.e. initial SOC, DOD, and C-rate distribution are representative of a typical HEV application. This current profile was repeated to cycle the cell at $45^{\circ} \mathrm{C}$ until it reached end-of-life (EOL), which was defined as $20 \%$ of cyclable capacity loss. As shown in Fig. 6 (b), the initial SOC was 
(a)

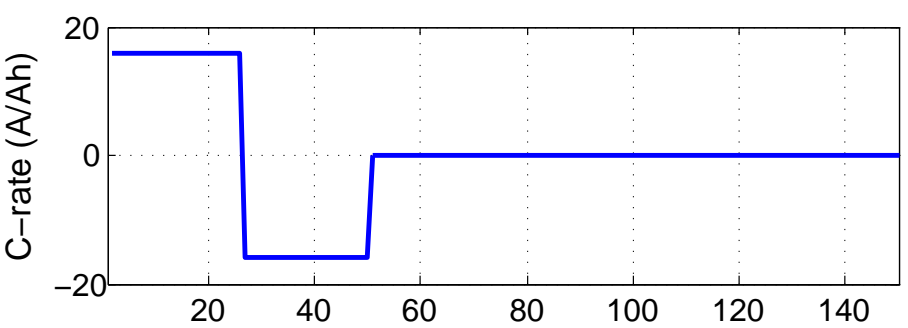

(d)

(b)

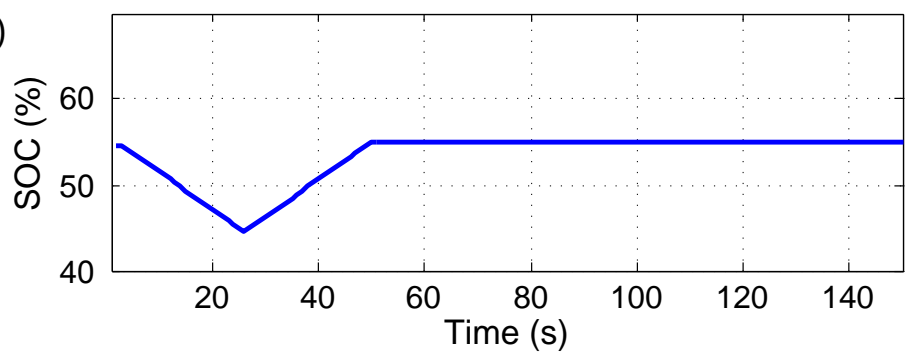

(c)
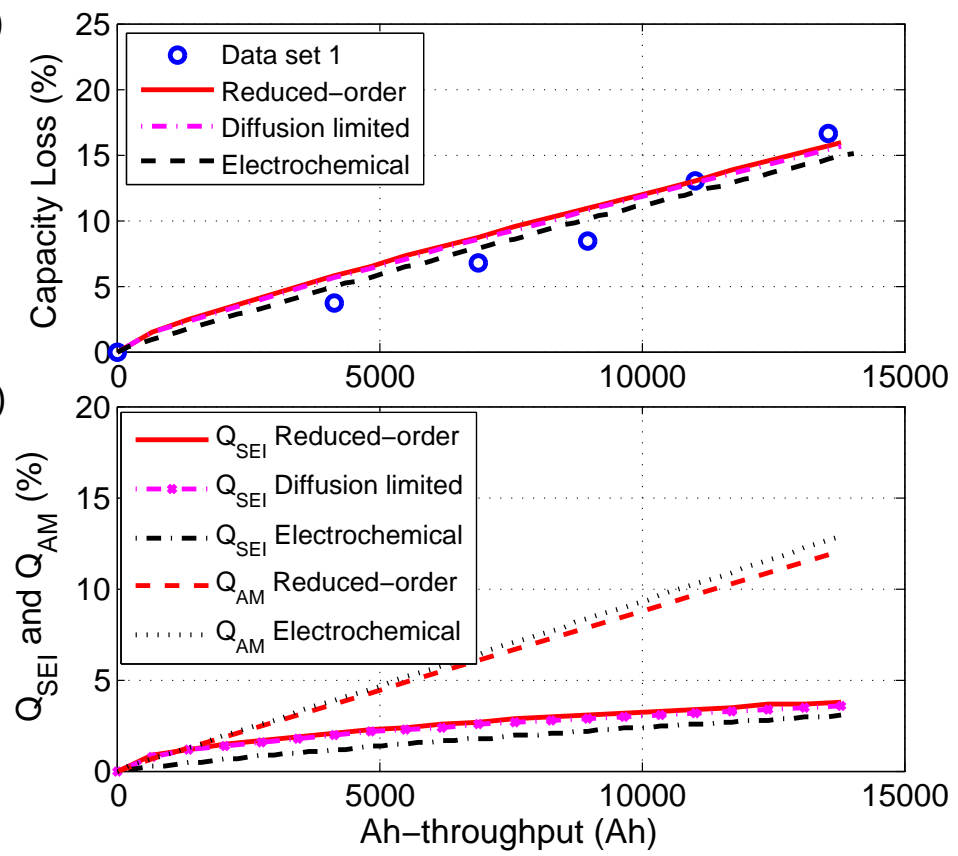

Figure 5: Model validation against Data Set 1 [22]. (a) shows the current profile to cycle the cell between $45 \%$ to $55 \%$ SOC. Ah-throughput per cycle $=$ Cell capacity $\times 2 \times$ DOD $=0.46$ Ah. (b) shows the corresponding SOC profile predicted by the pseudo-2D electrochemical model. (c) shows the simulation results vs. experimental data. (d) shows the $\mathrm{Q}_{\mathrm{SEI}}$ and $\mathrm{Q}_{\mathrm{AM}}$ predicted by reduced-order and pseudo-2D electrochemical models. 
$66 \%$, and SOC varied from $60 \%$ to $67 \%$ as the cell was cycled. The sub-model capacity loss, $\mathrm{Q}_{\mathrm{SEI}}$ and $\mathrm{Q}_{\mathrm{AM}}$, predicted by the three models is shown in Fig. 6 (d).

\subsection{Experimental data set 3-Representative of an electric vehicle (EV) cycle}

A comparison of experimental data, which is collected by cycling the cell with a current profile consistent with an EV application 21, and simulation results of all three models are shown in Fig. 7 (b). At $25^{\circ} \mathrm{C}$, the simulation results from the reduced-order and pseudo-2D electrochemical models correlate well with the experimental data. The diffusion limited model slightly underpredicts the capacity loss. At $45^{\circ} \mathrm{C}$, the electrochemical model shows very good agreement and the reduced-order model shows a slight underprediction compared with the pseudo-2D electrochemical model. Significantly increased percentage error (about 27\%) of the diffusion limited model is observed at $45^{\circ} \mathrm{C}$ as compared to $25^{\circ} \mathrm{C}$. The error of the diffusion limited model is mainly due to the underpreditcion of $\mathrm{Q}_{\mathrm{SEI}}$ (see Fig. 7 (c)), as the average SOC (around 90\%) for this dynamic EV cycle, as shown in Fig.7(a), is notably higher than the average SOC $(\sim 45 \%)$ of the diffusion limited model calibration data. This highlights the limitations of the diffusion limited sub-model for predicting capacity loss due to SEI layer growth.

The sub-model capacity loss, $\mathrm{Q}_{\mathrm{SEI}}$ and $\mathrm{Q}_{\mathrm{AM}}$, predicted by the three models is shown in Fig. 7 (c). Note that this drive cycle includes a large rest period of $2400 \mathrm{~s}$, and the overall C-rate of the dynamic current cycle is less than $2.5 \mathrm{C}$. As such, the capacity loss due to SEI layer growth (which is primarily calendardriven), $\mathrm{Q}_{\mathrm{SEI}}$, is slightly larger than that one due to active material loss, $\mathrm{Q}_{\mathrm{AM}}$. 
(a)

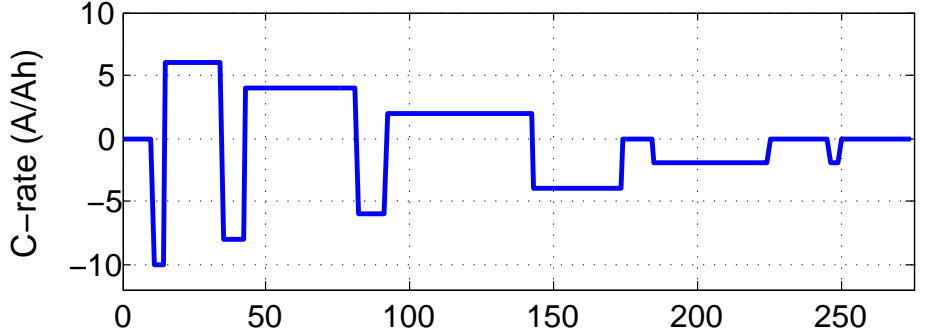

(b)

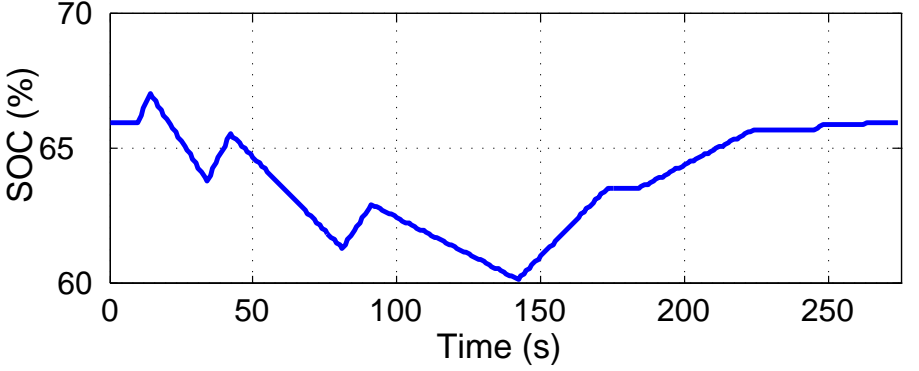

(d)
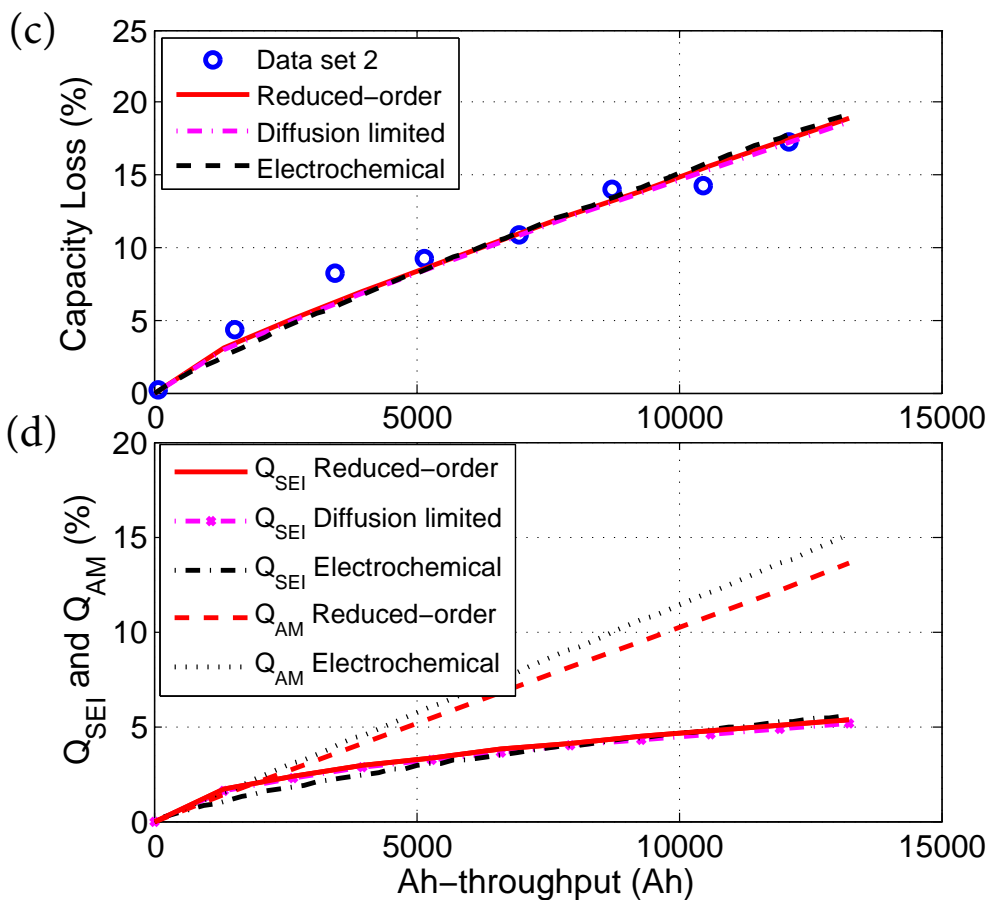

Figure 6: Model validation against Data Set 2 [20]. (a) shows the current profile representative of actual HEV operation. Sign convention: positive is discharging the battery, negative is charging the battery. Ah-throughput per cycle $=0.48 \mathrm{Ah}$. (b) shows the corresponding SOC profile predicted by the pseudo-2D electrochemical model. (c) shows the simulation results vs. experimental data set 2 [20. (d) shows the $\mathrm{Q}_{\mathrm{SEI}}$ and $\mathrm{Q}_{\mathrm{AM}}$ predicted by reduced-order and pseudo-2D electrochemical models. 

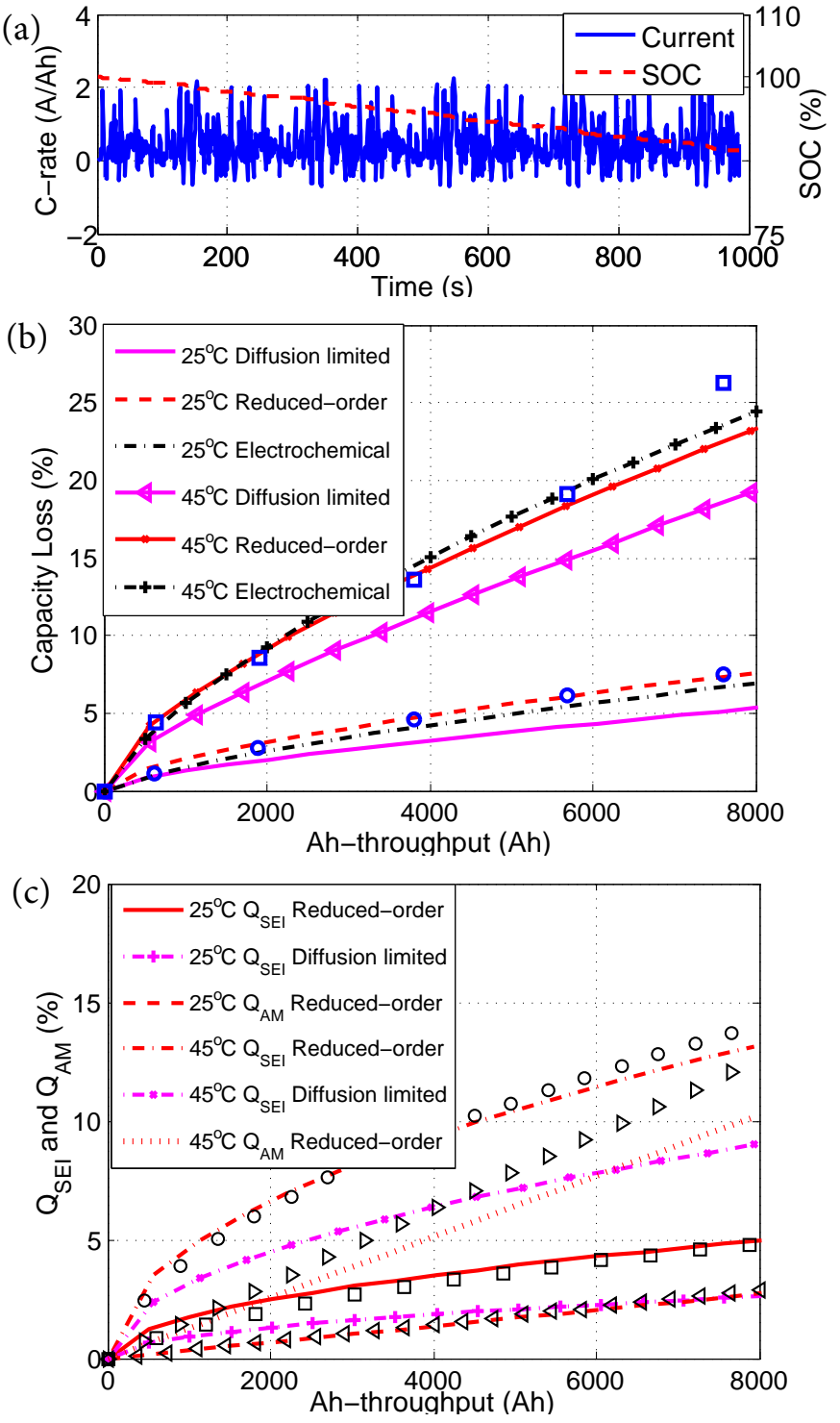

Figure 7: Model validation results against Data Set 3 21. In this experiment, the cell was first charged with a CC-CV protocol ( $0.5 \mathrm{C}$ current for constant-current part) up to $3.6 \mathrm{~V}$, after which it was left to rest for $30 \mathrm{~min}$, then discharged down to $3 \mathrm{~V}$ using the dynamic current profile shown in (a), and followed by a 10 min rest period. (b) shows the simulation result vs experimental result under EV drive cycle [21]. (c) shows the capacity loss due to SEI and active material loss. Left-pointing and right-pointing triangles represent the capacity loss due to active material loss predicted by the pseudo-2D electrochemical model for $25^{\circ} \mathrm{C}$ and $45^{\circ} \mathrm{C}$ respectively. Squares and circles are the capacity loss due to SEI layer growth predicted by the pseudo- $2 \mathrm{D}$ electrochemical model for $25^{\circ} \mathrm{C}$ and $45^{\circ} \mathrm{C}$ respectively. 


\subsection{Experimental data set 4 - Low C-rate cycle under varying temperatures} temperatures are compared with the experimental data, which is the result of a low C-rate $(0.5 \mathrm{C})$ testing under different temperature conditions [3]. The reduced-order and pseudo-2D electrochemical models show good agreement with the experimental data under different temperatures. The diffusion limited model underpredicts the capacity loss, mainly because of the underprediction of $\mathrm{Q}_{\mathrm{SEI}}$ as compared with the other two models, which is clearly seen from Fig. 8 (c). The lack of reduction kinetics information in the diffusion limited model makes it not generalizable for the operation conditions outside the calibration range. Experimental data from all DODs are included together for each temperature, increases, for $45^{\circ} \mathrm{C}$ and $60^{\circ} \mathrm{C}$, Q $\mathrm{SEI}$ becomes more significant as compared to $\mathrm{Q}_{\mathrm{AM}}$. Clearly temperature has more impact on $\mathrm{Q}_{\mathrm{SEI}}$ than $\mathrm{Q}_{\mathrm{AM}}$.

The maximum percentage error at the end point of the experimental data, predicted by each of the model across all four data sets, are summarized in Table

315 5. As shown, the pseudo-2D electrochemical model has a percentage error of $9 \%$ or less for all four data sets. The simplified reduced-order model performs similarly as compared with the pseudo-2D electrochemical model. Ignoring the reduction kinetics of the reduced-order model leads to a diffusion limited SEI layer growth model that has been presented in prior literature and a further 2 this paper, as it does not capture the impact of SOC and current on SEI layer growth. The diffusion limited model exhibits a percentage error less than $10 \%$ only when the data set is very similar to that used in the calibration process 

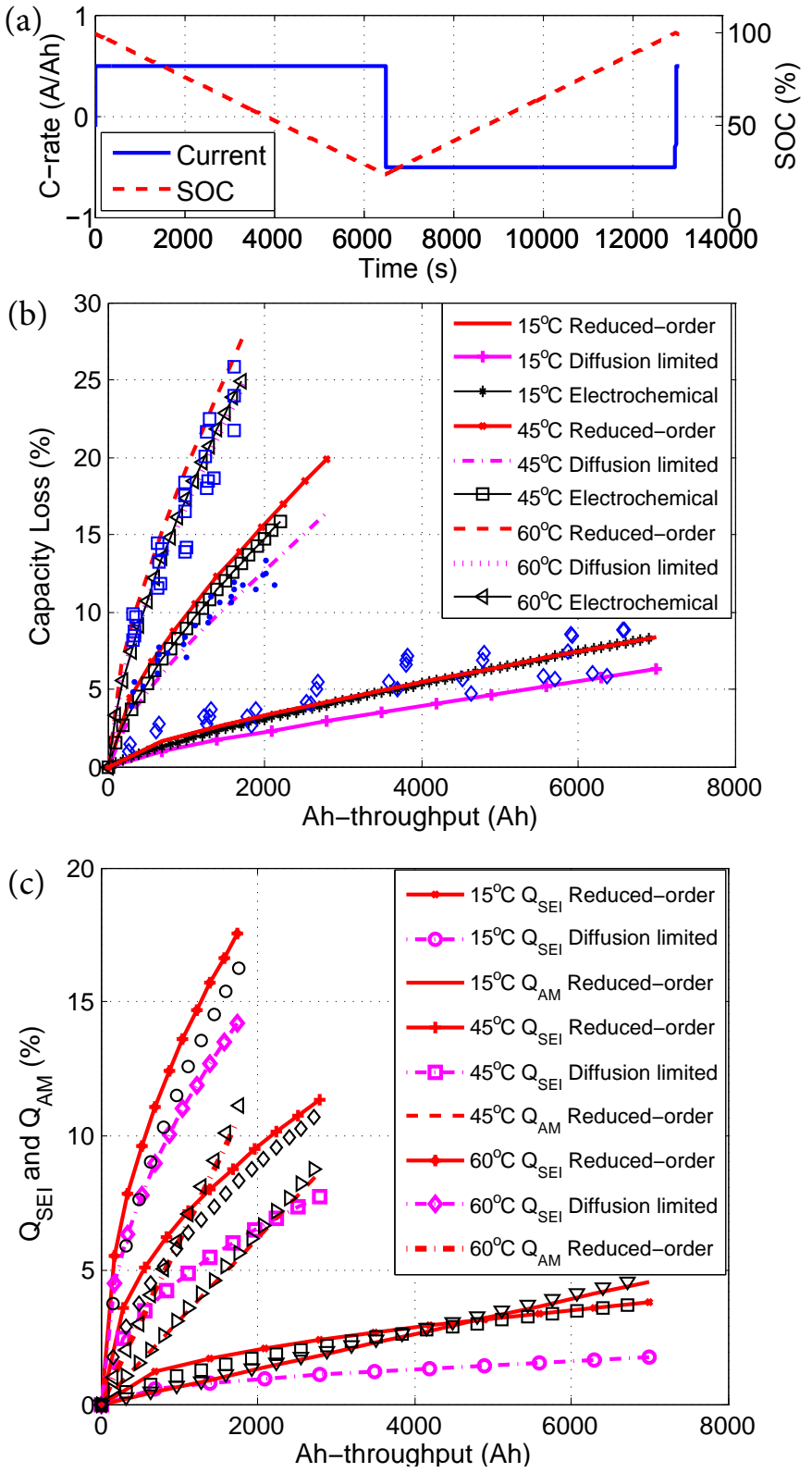

Figure 8: Model validation results against Data Set 4. (a) shows the current profile and SOC implemented in [3]. The cell was charged with a CC-CV protocol and discharged with a $0.5 \mathrm{C}$ constant current. (b) shows the experimental capacity loss measured at three different temperatures $\left(15^{\circ} \mathrm{C}, 45^{\circ} \mathrm{C}\right.$, and $\left.60^{\circ} \mathrm{C}\right)$, and corresponding simulation results. (c) shows the capacity loss due to SEI and active material loss. All DOD cases are combined in experimental data. 
325 (Data Set 1 and 2), otherwise the error is more than $20 \%$.

Table 5: Model comparison for the accuracy (in percentage error)

\begin{tabular}{cccc}
\hline Data Sets & Electrochemical & Reduced-order & Diffusion limited \\
\hline Data Set $1[22]$ & $8.9 \%$ & $5.1 \%$ & $6.1 \%$ \\
Data Set $2[20]$ & $2.0 \%$ & $2.0 \%$ & $1.0 \%$ \\
Data Set $3[21]$ & $6.5 \%$ & $10.0 \%$ & $27.6 \%$ \\
Data Set $4[3]$ & $8.0 \%$ & $8.5 \%$ & $22.7 \%$ \\
\hline
\end{tabular}




\section{Summary and Conclusions}

A computationally efficient, generalizable, reduced-order degradation model for Li-ion battery graphite anodes is derived and validated. The model is based on salient physical loss mechanisms and differs from computationally efficient of widely accepted constitutive laws, includes no ad-hoc terms, and can predict degradation accurately across a wide range of operating conditions. More complex electrochemical models couple these same constitutive laws with Liion transport PDEs, and as such, capacity loss prediction using those models order degradation model proposed in this paper only requires lumped input information (current vs time and SOC vs time) to predict the capacity loss. The reduced-order model is calibrated for a single data set, and shown to be able to predict the capacity loss with less than a $10 \%$ error for four other experimental data sets with considerably different operating conditions. It allows quantification of the capacity loss due to SEI growth and/or active material loss separately. Overall, the proposed model is able to match the prediction capability of electrochemical models, with a $2400 \times$ reduction in computational complexity.

\section{Acknowledgments}

This work was funded by the U.S. Department of Energy, Purdue University and Cummins Inc. through the Hoosier Heavy Hybrid Center of Excellence. The authors are grateful to Dr. Kandler Smith, Dr. C. Delacourt and Dr. M. Safari for sharing the EV drive cycle data. The authors would also like to acknowledge and Gary Parker from Cummins Inc. 


\section{Appendix A.}

$$
\begin{aligned}
U_{n}^{\mathrm{OCP}}= & 0.7222+0.1387 z+0.0299 z^{1 / 2}-\frac{0.0172}{z}+\frac{0.0019}{z^{1.5}} \\
& +0.2808 \exp (0.90-15 z)-0.7984 \exp (0.4465 z-0.4108)
\end{aligned}
$$

here $z=$ SOC.

\section{Appendix B.}

The diffusion equations to solve are:

$$
\begin{aligned}
& \frac{\partial C_{\mathrm{S}}(x, t)}{\partial t}=D_{\mathrm{S}} \frac{\partial^{2} C_{\mathrm{S}}(x, t)}{\partial x^{2}} \\
& \frac{\partial C_{\mathrm{P}}(x, t)}{\partial t}=D_{\mathrm{P}} \frac{\partial^{2} C_{\mathrm{P}}(x, t)}{\partial x^{2}}
\end{aligned}
$$

where the initial conditions, and the boundary conditions are:

$$
\begin{gathered}
C_{\mathrm{S}}(x, 0)=C_{\mathrm{S}}^{*} \\
C_{\mathrm{P}}(x, 0)=0 \\
\lim _{x \rightarrow \infty} C_{\mathrm{S}}(x, t)=C_{\mathrm{S}}^{*} \\
\lim _{x \rightarrow \infty} C_{\mathrm{P}}(x, t)=0
\end{gathered}
$$

and the charge flux density boundary conditions for the charge continuity equation are:

$$
\begin{gathered}
-D_{\mathrm{S}}\left(\frac{\partial C_{\mathrm{S}}(x, t)}{\partial x}\right)_{x=0}=\frac{i_{s}}{n F} \\
D_{\mathrm{S}}\left(\frac{\partial C_{\mathrm{S}}(x, t)}{\partial x}\right)_{x=0}=-D_{\mathrm{P}}\left(\frac{\partial C_{\mathrm{P}}(x, t)}{\partial x}\right)_{x=0}
\end{gathered}
$$


Apply the Laplace transform and solve Eq. B.1

$$
\begin{gathered}
s \bar{C}_{\mathrm{S}}(x, s)-C_{\mathrm{S}}^{*}=D_{\mathrm{S}} \frac{d^{2} \bar{C}_{\mathrm{S}}}{d x^{2}} \\
\frac{d^{2} \bar{C}_{\mathrm{S}}(x, s)}{d x^{2}}-\frac{s}{D_{\mathrm{S}}} \bar{C}_{\mathrm{S}}(x, s)=-\frac{C_{\mathrm{S}}^{*}}{D_{\mathrm{S}}}
\end{gathered}
$$

360

$$
\bar{C}_{\mathrm{S}}(x, s)=\frac{C_{\mathrm{S}}^{*}}{s}+A(s) \exp \left[-\sqrt{\left(s / D_{\mathrm{S}}\right)} x\right]+B(s) \exp \left[\sqrt{\left(s / D_{\mathrm{S}}\right)} x\right]
$$

The semi-infinite limit Eq. (B.5) can be transformed to

$$
\lim _{x \rightarrow \infty} C_{\mathrm{S}}(x, s)=\frac{C_{\mathrm{S}}^{*}}{s}
$$

Thus, $B(s)$ must be zero. Therefore,

$$
\bar{C}_{\mathrm{S}}(x, s)=\frac{C_{\mathrm{S}}^{*}}{s}+A(s) \exp \left[-\sqrt{\left(s / D_{\mathrm{S}}\right)} x\right]
$$

Similarly, Eq. B.2 can be solved as

$$
\bar{C}_{\mathrm{P}}(x, s)=B(s) \exp \left[-\sqrt{\left(s / D_{\mathrm{P}}\right)} x\right]
$$

Substitution of Eqs. (B.13) and (B.14) into the Laplace transform of Eq. (B.8) gives

$$
-A(s) \sqrt{D_{\mathrm{S}} s}-B(s) \sqrt{D_{\mathrm{P}} s}=0
$$

${ }_{365}$ Thus, $B(s)=-A(s) \lambda$, where $\lambda=\sqrt{D_{\mathrm{S}} / D_{\mathrm{P}}}$. The final solution of the concentration is

$$
\begin{gathered}
\bar{C}_{\mathrm{S}}(x, s)=\frac{C_{\mathrm{S}}^{*}}{s}+A(s) \exp \left[-\sqrt{\left(s / D_{\mathrm{S}}\right)} x\right] \\
\bar{C}_{\mathrm{P}}(x, s)=-A(s) \lambda \exp \left[-\sqrt{\left(s / D_{\mathrm{P}}\right)} x\right]
\end{gathered}
$$


Note that $C_{\mathrm{S}}(0, t)$ and $C_{\mathrm{P}}(0, t)$ are linked together by $\eta_{s}$, which is defined as $\eta_{s}=E-E_{e q}$. At equilibrium state, the potential of the anode is described by the Nernst equation:

$$
E_{e q}=E^{0^{\prime}}+\frac{R T}{n F} \ln \left[\frac{C_{\mathrm{S}}^{*}\left(C_{\mathrm{Li}}^{*}\right)^{2}}{C_{\mathrm{P}}^{*}}\right]
$$

When the side reaction occurs in the direction of reduction, the potential becomes:

$$
E=E^{0^{\prime}}+\frac{R T}{n F} \ln \left[\frac{C_{\mathrm{S}}(0, t)\left(C_{\mathrm{Li}}(0, t)\right)^{2}}{C_{\mathrm{P}}(0, t)}\right]
$$

Rearrange Eqs. (B.18) and (B.19) and define:

$$
\theta=\frac{C_{\mathrm{S}}(0, t)}{C_{\mathrm{P}}(0, t)}=\frac{C_{\mathrm{S}}^{*}\left(C_{\mathrm{Li}}^{*}\right)^{2}}{C_{\mathrm{P}}^{*}\left(C_{\mathrm{Li}}(0, t)\right)^{2}} \exp \left(\frac{n F}{R T} \eta_{s}\right)
$$

Equations (B.16), (B.17) and (B.20) leads to

$$
\frac{C_{\mathrm{S}}^{*}}{s}+A(s)=-\lambda \theta A(s)
$$

Solve $A(s)$ and plug it into Eq. (B.16) and Eq. (B.17)

$$
\begin{gathered}
\bar{C}_{\mathrm{S}}(x, s)=\frac{C_{\mathrm{S}}^{*}}{s}-\frac{C_{\mathrm{S}}^{*} \exp \left[-\sqrt{\left(s / D_{\mathrm{S}}\right)} x\right]}{s(1+\lambda \theta)} \\
\bar{C}_{\mathrm{P}}(x, s)=\frac{\lambda C_{\mathrm{S}}^{*} \exp \left[-\sqrt{\left(s / D_{\mathrm{P}}\right)} x\right]}{s(1+\lambda \theta)}
\end{gathered}
$$

Take the Laplace transform of Eq. (B.7) and substitute the derivative of $C_{\mathrm{S}}$

$$
\bar{i}_{s}=-\frac{n F C_{\mathrm{S}}^{*} \sqrt{D_{\mathrm{S}}}}{\sqrt{s}(1+\lambda \theta)}
$$

Then the inversion produces the current-time response

$$
i_{s}(t)=-\frac{n F C_{\mathrm{S}}^{*} \sqrt{D_{\mathrm{S}}}}{\sqrt{\pi t}(1+\lambda \theta)}
$$

Similar to $4,9,14, C_{\mathrm{Li}}(0, t)$ is assumed to be equal to the bulk concentration of $\mathrm{Li}, C_{\mathrm{Li}}^{*}$. To further simplify the calibration process, $C_{\mathrm{S}}^{*}$ and $C_{\mathrm{P}}^{*}$ in $\theta$ are assumed to be constant and embedded in $\lambda$, thus:

$$
\lambda=\frac{C_{\mathrm{S}}^{*} \sqrt{D_{\mathrm{S}}}}{C_{\mathrm{P}}^{*} \sqrt{D_{\mathrm{P}}}}
$$




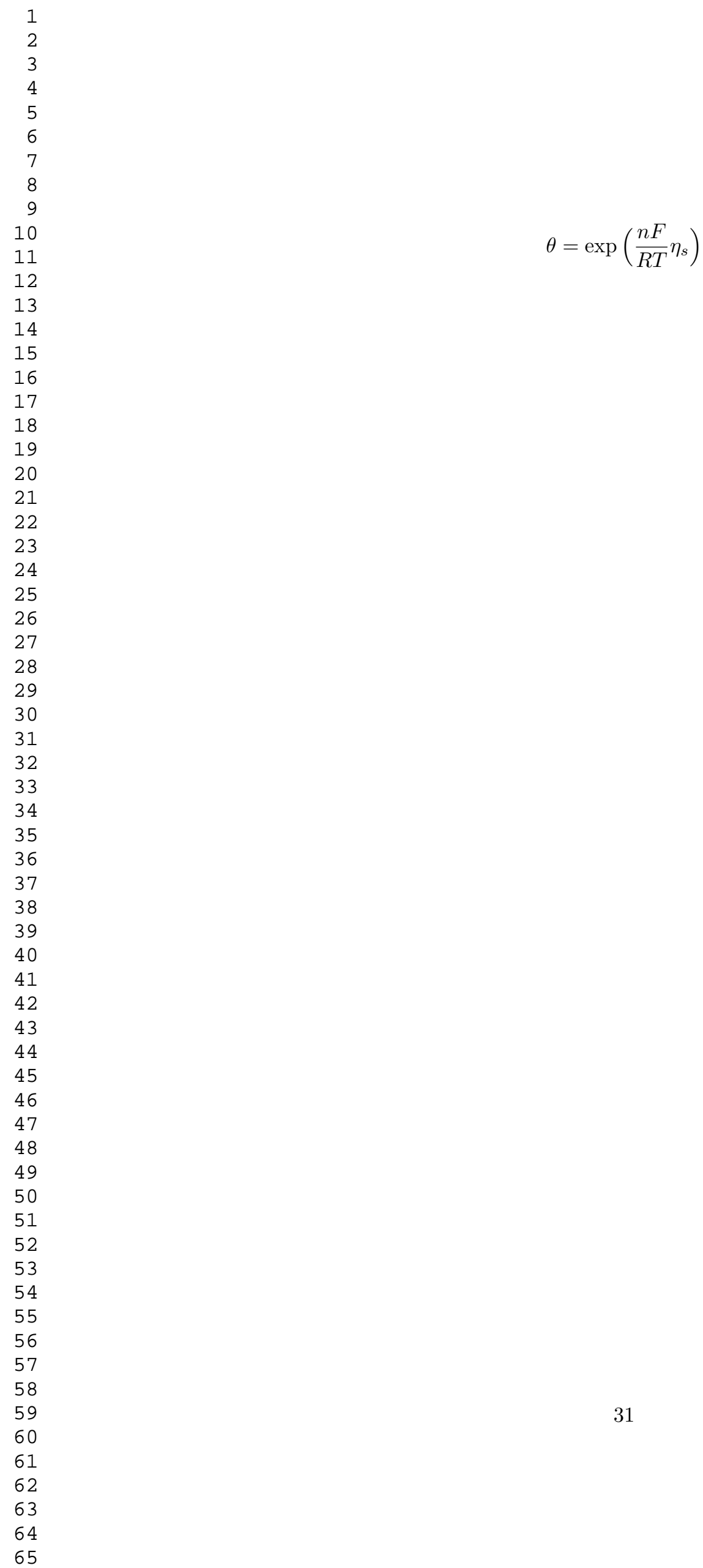




\section{References}

375 [1] B. Scrosati, J. Garche, Lithium batteries: Status, prospects and future, Journal of Power Sources 195 (9) (2010) 2419-2430.

[2] M. Oswal, J. Paul, R. Zhao, A comparative study of lithium-ion batteries, University of southern California (2010) 2419-2430.

[3] J. Wang, P. Liu, J. Hicks-Garner, E. Sherman, S. Soukiazian, M. Verbrugge, H. Tataria, J. Musser, P. Finamore, Cycle-life model for graphite-LiFePO4 cells, Journal of Power Sources 196 (8) (2011) 39423948. doi:10.1016/j.jpowsour.2010.11.134.

口 URL http://linkinghub.elsevier.com/retrieve/pii/ S0378775310021269

[4] M. Safari, C. Delacourt, Simulation-Based Analysis of Aging Phenomena in a Commercial Graphite / LiFePO 4 Cell 158 (12) (2011) 1436-1447. doi:10.1149/2.103112jes

[5] C. Delacourt, M. Safari, Life Simulation of a Graphite / LiFePO 4 Cell under Cycling 159 (8). doi:10.1149/2.049208jes.

390 400

[6] H. J. Ploehn, P. Ramadass, R. E. White, Solvent diffusion model for aging of lithium-ion battery cells, Journal of The Electrochemical Society 151 (3) (2004) A456-A462.

[7] A. Smith, J. C. Burns, X. Zhao, D. Xiong, J. Dahn, A high precision coulometry study of the sei growth in li/graphite cells, Journal of The Electrochemical Society 158 (5) (2011) A447-A452.

[8] M. B. Pinson, M. Z. Bazant, Theory of sei formation in rechargeable batteries: capacity fade, accelerated aging and lifetime prediction, Journal of the Electrochemical Society 160 (2) (2013) A243-A250.

[9] T. Coupled, B. Model, B. Packs, A Thermally Coupled Battery Model for System - level Analysis of Li - ion Battery Packs User s Manual. 
[10] E. Prada, D. Di Domenico, Y. Creff, J. Bernard, V. Sauvant-Moynot, F. Huet, A simplified electrochemical and thermal aging model of lifepo4graphite li-ion batteries: Power and capacity fade simulations, Journal of The Electrochemical Society 160 (4) (2013) A616-A628.

[11] P. Ramadass, B. Haran, P. M. Gomadam, R. White, B. N. Popov, Development of first principles capacity fade model for li-ion cells, Journal of the Electrochemical Society 151 (2) (2004) A196-A203.

[12] K. Smith, M. Earleywine, E. Wood, J. Neubauer, A. Pesaran, Comparison

1. of Plug-In Hybrid Electric Vehicle Battery Life Across Geographies and Drive Cycles doi : 10.4271/2012-01-0666. URL http://papers . sae.org/2012-01-0666/

[13] S. B. Peterson, J. Apt, J. Whitacre, Lithium-ion battery cell

a degradation resulting from realistic vehicle and vehicle-to-grid utilization, Journal of Power Sources 195 (8) (2010) 2385-2392. doi:10.1016/j.jpowsour.2009.10.010.

प URL http://linkinghub.elsevier.com/retrieve/pii/ S0378775309017443

[14] M. Safari, M. Morcrette, A. Teyssot, C. Delacourt, Multimodal PhysicsBased Aging Model for Life Prediction of Li-Ion Batteries, Journal of The Electrochemical Society 156 (3) (2009) A145. doi:10.1149/1.3043429.

URL http://jes.ecsdl .org/cgi/doi/10.1149/1.3043429

[15] R. P. Ramasamy, J.-W. Lee, B. N. Popov, Simulation of capacity loss in carbon electrode for lithium-ion cells during storage, Journal of power sources 166 (1) (2007) 266-272.

${ }_{425}$ [16] A. J. Bard, L. R. Faulkner, Electrochemical methods: fundamentals and applications, Vol. 2, Wiley New York.

[17] K. Smith, C.-Y. Wang, Power and thermal characterization of a lithium-ion 
battery pack for hybrid-electric vehicles, Journal of power sources 160 (1) (2006) 662-673.

430

[18] P. Arora, M. Doyle, A. S. Gozdz, R. E. White, J. Newman, Comparison between computer simulations and experimental data for high-rate discharges of plastic lithium-ion batteries, Journal of power Sources 88 (2) (2000) 219231.

[19] S. Santhanagopalan, Q. Guo, P. Ramadass, R. E. White, Review of models 158 (10). doi:10.1149/1.3614529.

[22] S. C. Nagpure, Multi-scale Characterization Studies of Aged Li-ion Battery Materials for Improved Performance. 